$\stackrel{W}{=}$

Global journals Inc.

की

\title{
Clinical Outcome, Return to Pre-Injury Activities and Patients Satisfaction after Open ACL Reconstruction and Arthroscopic Reconstruction; An Experience from a Developing Country
}

By Prof. Shehzad Javed, DR. Muhammad Farrukh Bashir, Dr. Zubair Khalid,

Dr. Umair Ahmed \& Prof Amer Aziz

Abstract-Introduction: There are two techniques for reconstruction of anterior cruciate ligament (ACL), open technique and arthroscopic assisted technique. Arthroscopic assisted technique has many advantages over open procedure but it needs more expertise and cost comparatively. The objective of this study is to identify the clinical outcomes on basis of lyshlomknee score (LKS) system and find out patients satisfaction after performing both procedures in two groups separately.

Material and Methods: Retrospective analysis of 600 patients undergoing open ACL reconstruction and arthroscopic reconstruction from 2005 to 2018 was done, at the Department of Orthopaedics, Ghurki Hospital, Lahore. We included all those patients who were 18 to 45 years of age and had at least 1 year follow up.

Keywords: anterior cruciate ligament (ACL), open technique, arthroscopic assisted technique, lyshlomknee score (Iks), satisfaction.

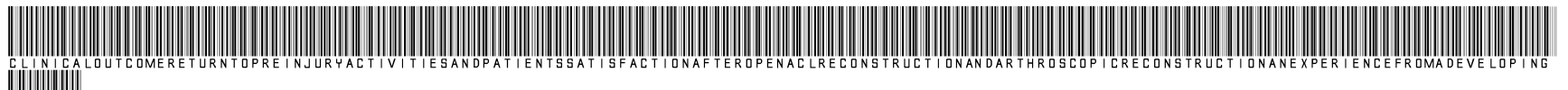

Strictly as per the compliance and regulations of:

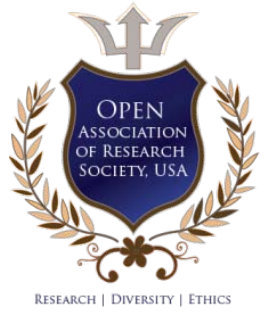

(c) 2020. Prof. Shehzad Javed, DR. Muhammad Farrukh Bashir, Dr. Zubair Khalid, Dr. Umair Ahmed \& Prof Amer Aziz. This is a research/review paper, distributed under the terms of the Creative Commons Attribution-Noncommercial 3.0 Unported License http://creativecommons.org/licenses/by-nc/3.0/), permitting all non-commercial use, distribution, and reproduction in any medium, provided the original work is properly cited. 


\title{
Clinical Outcome, Return to Pre-Injury Activities and Patients Satisfaction after Open ACL Reconstruction and Arthroscopic Reconstruction; An Experience from a Developing Country
}

\author{
Prof. Shehzad Javed ${ }^{\alpha}$, DR. Muhammad Farrukh Bashir ${ }^{\circ}$, Dr. Zubair Khalid ${ }^{\rho}$, Dr. Umair Ahmed ${ }^{\omega}$ \\ \& Prof. Amer Aziz
}

Abstract- Introduction: There are two techniques for reconstruction of anterior cruciate ligament (ACL), open technique and arthroscopic assisted technique. Arthroscopic assisted technique has many advantages over open procedure but it needs more expertise and cost comparatively. The objective of this study is to identify the clinical outcomes on basis of lyshlomknee score (LKS) system and find out patients satisfaction after performing both procedures in two groups separately.

Material and Methods: Retrospective analysis of 600 patients undergoing open $\mathrm{ACL}$ reconstruction and arthroscopic reconstruction from 2005 to 2018 was done, at the Department of Orthopaedics, Ghurki Hospital, Lahore. We included all those patients who were 18 to 45 years of age and had at least 1 year follow up. Amongst these patients, 500 patients underwent open $A C L$ reconstruction while 100 had arthroscopic reconstruction. Means along with standard deviation were calculated for the lysholm scoring between patients undergoing $\mathrm{ACL}$ reconstruction or arthroscopic reconstruction. Chi square test was applied to compare the qualitative variables like gender, while remaining quantitative variables like age, duration of follow up and LKS were compared using t-test for any significant difference in between both the groups. $P$ value less than or equal to 0.05 was considered as statistically significant.

Results: Out of 600 patients, there were 554 (92.3\%) male and $46(7.7 \%)$ female. Mean age of the patients was noted to be $30.2 \pm 4.3$ years. Overall, mean duration of follow up was noted to be 12 months. Overall, there was no significant difference in between both the groups in terms of gender, age. In open ACL patients, mean LKS was noted to be $82.78 \pm 14.78$ whereas in arthroscopic reconstruction group, mean LKS was noted to be $90.88 \pm 13.22$ while the difference between the two groups was statistically significant.

Conclusion: Majority of our patients were male. Although both studied procedures got good overall LKS but patients following arthroscopic reconstruction had significantly better LKS in comparison to open ACL reconstruction.

Author a: Professor, Department of Orthopaedics, Ghurki Hospital Lahore.e-mail: docshazi@gmail.com

Author б: Assistant Professor, Department of Orthopaedics, Ghurki Hospital Lahore.

Author p: Department of Orthopaedics, Ghurki Hospital Lahore, Pakistan.e-mail: zubair.khalid262@gmail.com

Author w: Senior Registrar, Department of Orthopaedic, Ghurki Hospital Lahore, Pakistan. e-mail: ravian.444@gmail.com

Author ¥: Chairman Orthopaedic Department, Ghurki Trust Teaching Hospital Lahore.
Keywords: anterior cruciate ligament (ACL), open technique, arthroscopic assisted technique, lyshlomknee score (Iks), satisfaction.

\section{InTRODUCTION}

- The Anterior Cruciate Ligament (ACL) has a major role in normal working of knee ${ }^{1}$. Rupture of $A C L$ is one of the most common diagnosis in young patients either due to RTA or sports trauma. Reconstruction of the ACL allows the patient to resume sporting activities and prevents damage in meniscus and articular cartilage in turn reducing chances of arthritis. ${ }^{2-4}$ There are two techniques for reconstruction of $A C L$, open technique and arthroscopic assisted technique. Arthroscopic assisted technique has many advantages over open procedure but it needs more expertise and cost comparatively. Currently, ACL reconstruction is most often performed using an arthroscopic assisted technique. ${ }^{5}$

Literature is deficient of $A C L$ reconstruction data in developing countries. In developing countries like Pakistan, cost is the major issue. Athroscopic assisted $A C L$ reconstruction is more expensive than open procedure. There is no large data available for such population which shows the clinical outcome after open \& arthroscopic ACL reconstruction. The objective of this study is to identify the clinical outcomes on basis of lyshlomknee score (LKS) system and find out patients satisfaction after performing both procedures in two groups separately.

\section{il. Material \& Methods}

Retrospective analysis of 600 patients undergoing open $A C L$ reconstruction and arthroscopic reconstruction from 2005 to 2018 was done at Department of Orthopaedics, Ghurki hospital, Lahore. We included all those patients who were 18 to 45 years of age and had at least 1 year follow up. Amongst these patients, 500atients underwent open $A C L$ reconstruction while 100 had arthroscopic reconstruction.

Lysholm scoring questionnaire as shown in Firgure- $1^{6,7}$ was adopted and enquired from all the 
patients. Face to face interview was done with all the study participants. If the patient stated that he/she did not understand the question properly, more explanation regarding that particular question was given until the patient understood what he/she was asked. All the study participants were invited to hospital. All those participants who found to be in the clinically stable state, were excluded from this study. All those cases that had any new related injury after $\mathrm{ACL}$ reconstruction or arthroscopic reconstruction were also excluded from the study. Patients who had evidence of clinical and radiological degenerative change in the knee were also excluded.A standard script was followed for all the interviews to maintain a level of consistency. All the ethical standards written in "The Declaration of Helsinki 1964 "8 and its later amendments were fully followed in this study.

Means along with standard deviation were calculated for the lysholm scoring between patients undergoing $\mathrm{ACL}$ reconstruction or arthroscopic reconstruction. Chi square test was applied to compare the qualitative variables like gender, while remaining quantitative variables like age, duration of follow up and LKS were compared using t-test for any significant difference in between both the groups. $P$ value less than or equal to 0.05 was considered as statistically significant.
SECTION 1 - LIMP

$\square$ I have no limp when I walk. (5)

$\square$ I have a slight or periodical limp when I walk. (3)

I have a severe and constant limp when I walk. (0)

\section{SECTION 2 - Using cane or crutches \\ $\square$ I do not use a cane or crutches. (5) \\ $\square$ I use a cane or crutches with some weight-bearing. (2) \\ $\square$ Putting weight on my hurt leg is impossible. (0)}

\section{SECTION 3 - Locking sensation in the knee}

$\square$ I have no locking and no catching sensation in my knee. (15)

$\square$ I have catching sensation but no locking sensation in my knee. (10)

$\square$ My knee locks occasionally. (6)

My knee locks frequently. (2)

My knee feels locked at this moment.. (0)

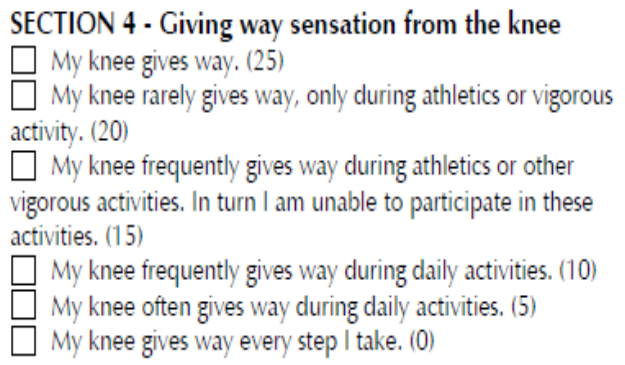

\section{SECTION 5 - PAIN}

$\square$ I have no pain in my knee. (25)

$\square$ I have intermittent or slight pain in my knee during vigorous activities. (20)

$\square$ I have marked pain in my knee during vigorous activities. (15)

$\square$ I have marked pain in my knee during or after walking more than 1 mile. (10)

$\square$ I have marked pain in my knee during or after walking less than 1 mile. (5)

$\square$ I have constant pain in my knee. (0)

\section{SECTION 6 - SWELLING}

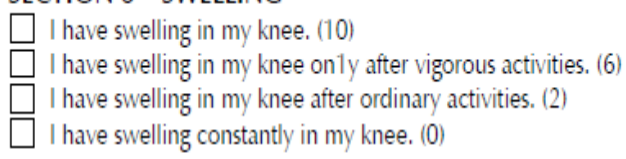

\section{SECTION 7 - CLIMBING STAIRS}

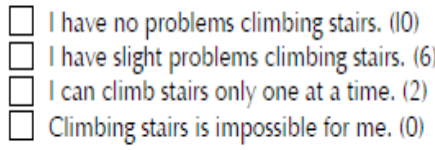

\section{SECTION 8 - SQUATTING}

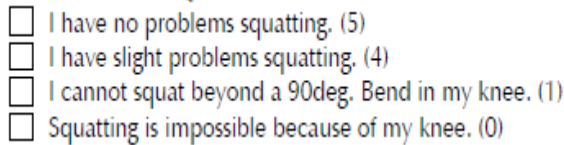

Total: $/ 100$

\section{Figure 1: Lysholm knee score $(\mathrm{LKS})^{6,7}$}

\section{RESUlTS}

In this study, a total of 600 patients were included among these majority 554 (92.3\%) were male and $46(7.7 \%)$ were female patients with an average age of $30.2 \pm 4.3$ years. Overall, mean duration of follow up was noted to be $21.4 \pm 5.6$ months.

Table 1 showed the demographic profile of patients underwent two different surgical procedures, in group 1 500involvedunderwent open ACL among these93\% were males and $7 \%$ were females with an average age of $30.27+4.2$ years and follow up duration of 12 months as compared to group 2 where the patients of arthroscopic reconstruction took part in the study $n=100$ among these $89 \%$ were male participants and $11 \%$ were females with an average age of $29.57+4.7 y e a r s$ and had the follow up duration of 12 months. The study reveals that there was no significant difference in between both the groups in terms of gender, age but duration of follow up were different in both groups as ( $p$ value $\leq 0.05$ ).

Table 2 demonstrates the functional outcome of patients using Lysholm knee score among two groups of patients. In open ACL patients, majority $55 \%$ patients 
reported excellent outcome, $30 \%$ with good outcome with an average score of $82.78 \pm 14.78$ whereas in arthroscopic reconstruction group, majority reported excellent functional outcome as $90 \%$ reported excellent functional outcome and $2 \%$ with poor outcome with an average score of $90.88 \pm 13.22$ and statistically significant difference were obtained in the mean LKS score in both groups as ( $p$-value $\leq 0.05)$.

Table 1: Demographic Profile of patients underwent two surgical Intervention $(n=600)$

$\mathrm{n}(\%)$ or Mean \pm S.D

\begin{tabular}{|c|c|c|c|}
\hline Characteristics & $\begin{array}{l}\text { Open ACL } \\
(n=500)\end{array}$ & $\begin{array}{l}\text { Arthroscopic Reconstruction } \\
\qquad(n=100)\end{array}$ & p-value \\
\hline $\begin{array}{l}\text { Male } \\
\text { Female }\end{array}$ & $\begin{array}{c}465(93) \\
35(7)\end{array}$ & $\begin{array}{l}89(89) \\
11(11)\end{array}$ & 0.2136 \\
\hline Age (mean+SD) & $30.27+\_4.2$ & $29.57+\_4.7$ & 0.1366 \\
\hline Duration of follow up & 12 & 12 & \\
\hline
\end{tabular}

Table 2: Functional outcome of Patientsusing LKS score among two Groups( $\mathrm{n}=600)$ $n(\%)$

\begin{tabular}{|c|c|c|c|c|c|}
\hline \multirow[b]{2}{*}{ Outcome } & \multicolumn{2}{|c|}{$\begin{array}{c}\text { Group } 1 \\
\text { Open ACL }\end{array}$} & \multicolumn{2}{|c|}{$\begin{array}{c}\text { Group } 2 \\
\text { Arthroscopic ACL }\end{array}$} & \multirow[b]{2}{*}{ p-value } \\
\hline & $n=500$ & Mean $\pm S . D$ & $n=100$ & Mean $\pm S . D$ & \\
\hline Excellent & $450(90)$ & & $55(55)$ & & \\
\hline Good & $20(4)$ & $82.78 \pm 14.78$ & $30(30)$ & $90.88 \pm 13.22$ & $* 0.021$ \\
\hline Fair & $20(4)$ & & $10(10)$ & & \\
\hline Poor & $10(2)$ & & $5(\%)$ & & \\
\hline
\end{tabular}

${ }^{*} p$-Value $\leq 0.05$ considered to be significant

\section{Discussion}

Open ACL reconstruction and arthroscopic reconstruction are not new as lots of literature is available about these two but the debate regarding which approach is better is still going on. ${ }^{9}$ In this retrospective analysis, our objective was to compare LKS scores following ACL reconstruction and arthroscopic reconstruction, and comparing with each other.

Overall, $92.3 \%$ of the patients in our findings were male. It has been a well established fact that male population is more exposed to road accidents and outdoor activities, ${ }^{10,11}$ this could be the major reason why significantly more male are reported involving reconstruction procedures.
Quite a few systems have been developed in the recent years evaluating pre as well as post operative condition of knee area. Different protocols are available but most are based on functional as well clinical evaluations. O'Donoghue is known to be the $1^{\text {st }}$ to apply scale system aiming to evaluate post operative results. ${ }^{12}$ Our objective was to compare the post operative outcome of ACL reconstruction and arthroscopic reconstruction in knee injuries based on follow up (at least 1 year). Various methods were considered aiming to evaluate knee region. We got attracted to Lysholmknee scaling (LKS) score which is based on the modified Lysholm protocol and has been used extensively all around the world. LKS has also been noted to have high reliability, validity as well as responsiveness all over the world. ${ }^{13-17}$ This was the very 
reason that we adopted this scale and we are confident that translating results using such scale will further benefit larger proportions of our population.

In the present study, open ACL patients, mean LKS was noted to be 82.78 with a standard deviation of 14.78 whereas in arthroscopic reconstruction group, mean LKS was noted to be 90.88 with a standard deviation of 13.22 while the difference between the two groups was statistically significant. In a recent study conducted by $\mathrm{L}$. de Villiers ${ }^{18}$ to find out the prevalence of osteoarthritis in the knee in the long term after ACL reconstruction, 43 patients were evaluated as per LKS. Mean KLS score was noted to be 84.35 in those patients. These results are very similar to our findings where we noted mean KLS score to be 82.78 in our patients.

A study done by Kose $\mathrm{O}$ et $\mathrm{al}^{11}$ noted the mean LKS score to be 93.56 which is close to what we found in the present study. Overall mean follow up in that study was recorded to be 33.4 months which is quite higher in comparison to what we had in our findings. While comparing, open ACL reconstruction and arthroscopic reconstruction group, mean LKS was noted to be significantly higher in arthroscopic reconstruction patient showing overall better results of following this technique.

\section{Conclusion}

Majority of our patients were male. Although both studied procedures got good overall LKS but patients following arthroscopic reconstruction had significantly better LKS in comparison to open ACL reconstruction.

\section{References Références Referencias}

1. Kaipour AM, Murray MM. Basic science of anterior cruciate ligament injury and repair. Bone Joint Res. 2014; 3(2):20-31.

2. Quatman CE, Kiapour AM, Demetropoulos CK, Kiapour A, Wordeman SC, Levine JW, et al. Preferential loading of the ACL compared with the MCL during landing: a novel in sim approach yields the multiplanar mechanism of dynamic valgus during ACL injuries. Am J Sports Med 2014; 42: 177-186.

3. Tran TD, QuocLT. A cadaveric study on the anatomy of anterior cruciate ligament in Vietnamese adults. Asia-Pacific Journal of Sports Medicine, Arthroscopy, Rehabilitation and Technology 2018; 14:22-25.

4. Mansoori N, Behera C, Kalyanasundaram D, Marieswaran, Digge VK, Jhajhria SK, et al. Effect of preservation methods on tensile properties of human femur-ACL-tibial complex (FATC) - A cadaveric study on male subjects. Acta of
Bioengineering and Biomechanics 2018; 20(3): 3142.

5. Paschos NK, Howell SM. Anterior cruciate ligament reconstruction: principles of treatment. EFORT Open Rev. 2016 Nov; 1(11): 398-408.

6. Tegner $\mathrm{Y}$, Lysholm J. Rating systems in the evaluation of knee ligament injuries. ClinOrthopRelat Res. 1985;(198):43-9.

7. Mitsou A, Vallianatos P, Piskopakis N, Maheras S. Anterior cruciate ligament reconstruction by overthe-top repair combined with popliteus tendon plasty. J Bone Joint Surg Br. 1990 May; 72(3):398404.

8. WMA declaration of helsinki - ethical principles for medical research involving human subjects. Adopted by the 18th WMA General Assembly, Helsinki, Finland, June 1964.

9. Barzegar H, Mohseni M, Sedighi A, Shahsavari A, Mohammadpour H.Arthroscopically-assisted vs. open surgery in repairing anterior cruciate ligament avulsion. Pak J Biol Sci. 2011; 14(8):496-501.

10. Jomha NM, Pinczewski LA, Clingeleffer $A$, et al. Arthroscopic reconstruction of anterior cruciate ligament with patellar-tendon autograft and interference screw fixation. The results at seven years. J Bone Joint Surg (Br) 1999; 81:775.

11. Kose O, Deniz G, Ozcan H. A comparison of telephone interview versus on-site completion of Lysholm knee score in patients who underwent arthroscopic ACL reconstruction: are the results equivalent? Eur J OrthopSurgTraumatol2005; 25(6):1069-72.

12. O' Donoghue DH. An analysis of end results of surgical treatment of major injuries to the ligaments of the knee. J Bone Joint Surg Am. 1955; 37(1):113.

13. Peccin MN, Ciconelli R, Cohen M. Specific questionnaire for knee symptoms- The Lysholm knee scoring scale- Translation and validation into Portuguese. ActaOrtop Bras. 2006; 14(5): 268-72.

14. Bengtsson J, Mollborg J, Werner S. A study for testing the sensitivity and reliability of the Lysholm knee scoring scale. Knee Surg Sports TraumatolArthrosc. 1996; 4(1):27-31.

15. Briggs KK, Kocher MS, Rodkey WG, Steadman JR. Reliability, validity, and responsiveness of the Lysholm knee score and Tegner activity scale for patients with meniscal injury of the knee. J Bone Joint Surg Am. 2006; 88(4):698-705.doi: 10.2106/JBJS.E.00339.

16. Paxton EW, Fithian DC, Stone ML, Silva P. The reliability and validity of knee-specific and general instruments in assessing acute patellar dislocation outcomes. Am J Sports Med. 2003; 31(4):487-492.

17. Kocher MS, Steadman JR, Briggs KK, Sterett WI, Hawkins RJ. Reliability, validity, and responsiveness 
of the Lysholm knee scale for various chondraldisorders of the knee. J Bone Joint Surg Am. 2004; 86(6):1139-1145.

18. L. de Villers. Anterior cruciate ligament reconstruction: a long-term follow-up. Orthopaedic Proceedings 2018; 87-B (Supp-1). 